lange das Thier lebte, an der totalen Exstirpation der Labyrinthe zweifelten, andere die, weil sie an die specifische Energie der Endapparate glaubten, geneigt waren die gesebenen Bewegungen auf Schallreflexe des Acusticus olne begleitende bewusste Gehörsempfindungen zurückzuführen. Aber ich habe niemanden gefunden, dem nicht die von Bernstein am Schlusse seines Aufsatzes aufgestellte Hypothese einer unbeabsichtigten Hantreizung als absolut ausgeschlossen erschienen wäre. Auch Bernstein s neue Versuche halte ich, insoweit er sie zu Gunsten dieser Hypothese verwerthet, nicht für beweiskräftig, enthalte mich aber so lange einer näheren Kritik derselben, als mir nicht eigene weitere Beobachtungen zu Gebote stehen. Immerhin betrachte ich es als einen Fortschritt, dass sich B e rnste in von der Existenz der Schallreactionen labyrinthloser Thiere, nachdem er diese noch in seinem vorigen Aufsatze (Bd. 57. S. 477 dieses Archivs) für eine "arge Täuschung" erklärt hatte, nunmehr selbst überzengt hat.

(Aus dem physiologischen Institute in Bonn.)

\title{
Die quantitative Bestimmung von Fett in thierischen Organen.
}

\author{
Vorläufige Mittheilung \\ von
}

\section{Dr. phil. C. Dormeyer.}

Die Angaben in der physiologisch-chemischen Literatur lassen keinen Zweifel über die Zuverlässigkeit der bisherigen quantitativen Methode der Bestimmung von Fett in thierischen Organen aufkommen. Dieselbe besteht bekanntlich darin, dass das getrocknete Organ mit siedendem Aether mehrere Stunden extrahirt wird.

Im Gegensatz dazu hat sich nun herausgestellt, dass es dem Aether nur sehr schwer gelingt, das Fett ans den Zellen herauszaholen und die Menge des Aetherextractes abhängig ist von der mehr oder weniger feinen Beschaffenbeit des betreffenden Organpulvers und der Dauer der Extraction. 
Die angestellten Versuche haben Folgendes ergeben:

1. Wenn man in vacuo' getrocknetes und fein pulverisirtes Fleisch, und zwar sogar solches, das auf das sorgfältigste von sichtbarem Fett und auch vom Bindegewebe nach Möglichkeit befreit ist, so lange im Soxhlet'schen Apparat mit wasserfreiem Aether auszieht, bis nur noch ganz geringe Mengen von Fett erhalten werden, wozu etwa 100 Stunden genügen, so ist trotz alle dem je nach den Bedingungen noch $1 / 5-1 / 4$ der zu bestimmenden Fettmengen in dem Fleischpulver enthalten.

2. Denn nachdem das Fleischpulver aufs Neue mebrere Stunden im Mörser zerrieben worden ist, erhält man bei erneuter Extraction mit Aether noch beträchtliche Fettmengen. Fährt man mit der Extraction, ohne zu pulverisiren, so lange fort, bis wieder zu vernachlässigende Fettmengen gewonnen werden, so ist abermals das Fleischpulver nicht erschöpft. Pulverisirt man dasselbe wiederum sehr energisch mehrere Stunden, so erhält man bei der Extraction abermals nicht zu vernachlässigende Fettmengen. Wiederholte ich die Procedur des Pulverisirens wieder und wieder, so gelangte ich selbst nach fast 5 monatlicher täglicher Extraction keineswegs zur Erschöpfung des Pulvers. Es bleibt zu beachten, dass die höchste Feinheit des Fleischpulvers erst erzielt werden kann, nachdem demselben die grössere Menge des Fettes entzogen worden ist. Der so gewonnene Aetherextract bestand der Hauptsache nach aus Nentralfetten. Die iibrigen Bestandtheile: flüchtige und höhere Fettsäuren, Lecithin, Cholestearin und stickstoffhaltige Extractivstoffe waren darin in so geringer Menge vorhanden, dass man den Extract, ohne einen wesentlichen Fehler zu begehen, als Nentralfett in Rechnung ziehen darf.

3. Da es sich nach obigem als unausführbar herausstellte, durch Extraction mit Aether das getrocknete Fleisch an Fett zu erschöpfen, so wurde auf Vorschlag von Prof. P fl iu g e r das so monatelang extrahirte Pulver durch Magensaft verdaut. Die erhaltene Lösung wurde alsdann mit Aether ausgeschättelt. Es ergab sich dabei die auffällige Thatsache aus mehreren Versuchen, dass das Fleischpulver im Durehschnitt noch $0,75 \%$ Fett enthielt. Nach der Verseifung mit alkoholischem Kali ergab das erhaltene Fett die berechnete Menge an Fettsäuren.

Die analytischen Belege und die genane Angabe des Analysenganges werde ich in kurzer Zeit des Näheren mittheilen. 